Ann. Zootech., I963, $12(2)$, I25-I32.

\title{
COMPARAISON DE DIFFÉRENTES MÉTHODES DE MESURE DU POUVOIR DE RÉTENTION D'EAU DE LA VIANDE DE PORC. LIAISON AVEC LE PH
}

\author{
R. GOUTEFONGEA \\ Laboratoire de Recherches sur la Viande, \\ Centre national de Recherches zootechniques, Jouy-en-Josas (Seine-et-Oise)
}

\section{SOMMAIRE}

Deux méthodes de mesure du pouvoir de rétention d'eau de la viande de porc, l'une faisant appel à la compression, l'autre à la centrifugation, ont été comparées en utilisant 480 échantillons de muscle, provenant de 30 sujets de race Large White.

On a également comparé les résultats obtenus par la méthode de compression, en fonction du mode d'expression des résultats, ainsi que les relations entre le $\mathrm{pH}$ et le pouvoir de rétention d'eau mesuré par les différentes méthodes.

La méthode de centrifugation et celle de compression (cette dernière quand on mesure la perte de poids par pesée et qu'on la rapporte au poids frais) ont sensiblement la même variabilité et ont entre elles un coefficient de corrélation hautement significatif.

Les résultats obtenus par la méthode de centrifugation sont liés au pH plus étroitement que ceux obtenus par compression.

La mesure du pouvoir de rétention d'eau est utilisée pour apprécier la qualité de la viande; on cherche alors surtout à déterminer la force avec laquelle l'eaut est liée aux protéines musculaires, ou la quantité relative d'eau qui est liée fortement à ces protéines, afin de prévoir le comportement ultérieur de la viande : pertes à la conservation, à la cuisson, à la décongélation, facilités de conservation. En outre, ce pouvoir de rétention d'eau est en relation assez étroite avec l'aspect que présentera la viande au moment de sa commercialisation et sa mesure permet donc des prévisions en ce qui concerne son mode d'utilisation.

La mesure du pouvoir de rétention d'eau en valeur absolue peut difficilement se concevoir actuellement. En effet, on passe insensiblement d'une eau presque fixe à une eau presque libre (Hamburger et Mathe, I952). L'eau du muscle s'y trouve sous diverses formes. Des molécules d'eau sont liées chimiquement à des molécules organiques, ce qui constitue une eau fixe permanente dans la cellule. D'autres molécules d'eau sont retenues physiquement par les micelles au moyen de liaisons électrostatiques. Ces molécules sont disposées autour des micelles en couches concentriques et celles qui ont une position externe sont retenues avec moins de force. Il est donc impossible de faire une séparation stricte entre les deux formes d'eau. 
D'autres part, les différentes méthodes visant à séparer l'eau libre de l'eau liée, ou visant à déterminer la fraction libre de l'eau totale, ce qui revient au même, et qui sont basées sur des phénomènes de congélation, évaporation, absorption par l'alcool, donnent en général des résultats peu concordants (HAMBURGER et MATHE, I952).

Il n'est donc possible de parler d'eau liée et d'eau libre que dans des conditions d'extraction bien définies et précises. Chaque méthode d'extraction définissant une eau liée et une eau libre, seuls des résultats obtenus avec la même méthode, dans desconditions rigoureusement identiques, peuvent être comparés.

En soumettant des échantillons de viande au même ensemble de conditions. d'extraction et en mesurant la quantité d'eau exprimée, on peut dire que tel échantillon a plus ou moins d'eau libre que tel autre échantillon et, par suite, que le pouvoir de rétention d'eau de cette viande est plus ou moins élevé.

Diverses méthodes ont été proposées pour l'extraction de l'eau « libre " ou " peu. liée ", c'est-à-dire de la partie de l'eau qui se sépare facilement de la viande. Toutes. ces méthodes font appel soit à la centrifugation, soit à la compression.

WIERBICKI, KUNKLE et DEATHERAGE (I957), travaillant sur le bœuf, utilisent. la centrifugation à sec, c'est-à-dire en opérant sur un broyat de muscles obtenu sans. adjonction d'eau.

Par contre, SWIFT et BERMANN (1959), utilisent la centrifugation également, mais après adjonction d'eau au broyat. De même, Shermanis (I96I) pratique la centrifugation après adjonction d'eau.

Dans tous les cas, les résultats sont exprimés par rapport au poids frais en pourcentage d'eau extraite ou absorbée par la viande à l'issue de la centrifugation.

Les méthodes faisant appel à la compression sont les plus utilisées. Ces méthodessont toutes dérivées, avec des modifications plus ou moins importantes, de celle de GRAU et HAMm (r952) qui consiste à presser l'échantillon de viande sur un papierfiltre, la pression étant établie à la main à l'aide d'une presse à vis. Cette méthode a été reprise par différents auteurs, sans modifications, ou avec des modifications de détail visant en général à l'obtention d'une pression constante ou au moins indépendante de l'opérateur. Les caractéristiques de ces variantes sont rassemblées dans. le tableau I.

On peut aussi, selon les auteurs, noter des variations dans le mode d'expression des résultats.

GraU et HAMm (I953) considèrent la surface totale de la tache humide obtenue, puis la différence entre cette surface et celle de la viande après pression et même la. surface de la viande seule.

Wismer-PEDERSEN (I958) considère la différence entre poids de viande initial et final ; BRISKEY et al. (I959) utilisent la différence entre surface totale et surface de la viande. Wierbicki et Deatherage (I958) également.

Nous avons pris en considération à la fois la surface totale et la différence de poids (GoUTEFoNGEA, I960).

Les méthodes de mesure étant très nombreuses, il nous a semblé nécessaire de réaliser une étude comparative. Nous avons voulu comparer :

- les résultats obtenus avec une méthode faisant appel à la centrifugation et ceux obtenus avec une méthode faisant appel à la compression,

- les résultats obtenus par compression, selon leur mode d'expression. 
TABLEAU I

Caractéristiques des méthodes de mesure du pouvoir de rétention d'eau de la viande dérivées de la méthode de GRAU et HaMm (1952)

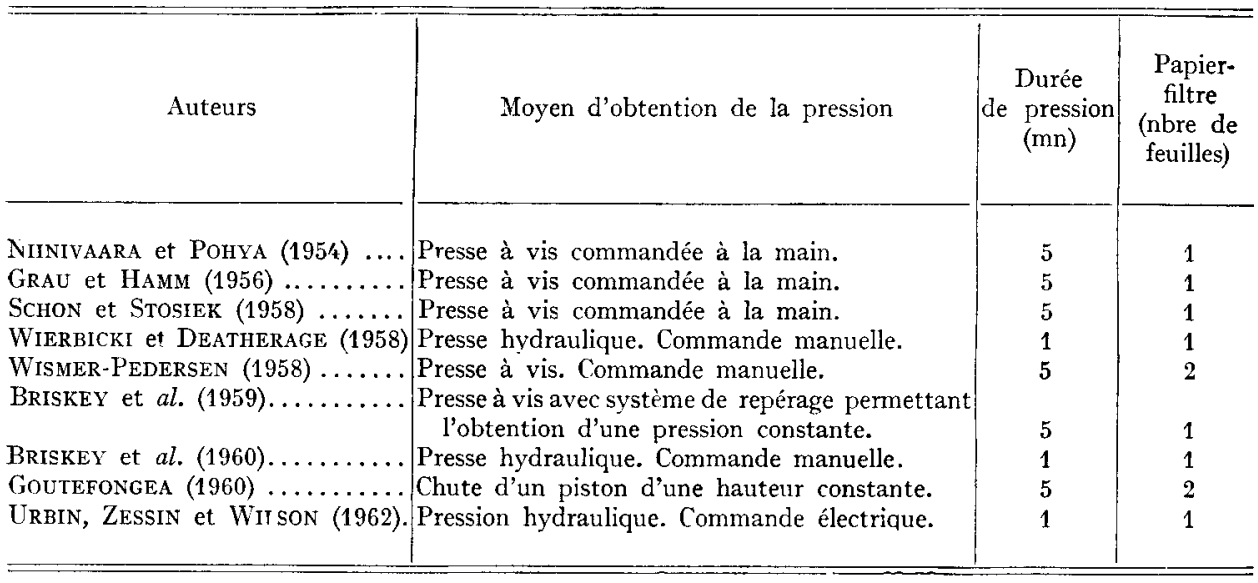

Nous avons également étudié la relation entre la mesure du pouvoir de rétention d'eau par ces différentes méthodes et le $\mathrm{pH}$ des échantillons.

\section{MATÉRIEL E'T TECHNIQUES}

Nous avons utilisé au total 480 échantillons de muscle de porcs provenant de 30 sujets de race Large White. La qualité variait de très bon à exsudatif et couvrait toute la gamme des qualités trouvées dans le commerce.

Toutes les déterminations étaient faites $24 \mathrm{~h}$ après l'abattage. La mesure du $\mathrm{pH}$ était faite avec un $\mathrm{pH}$ mètre EIL, type $23 \mathrm{AF}$ et une électrode duplex.

Les processus opératoires des méthodes de compression et centrifugation étaient les suivantes :

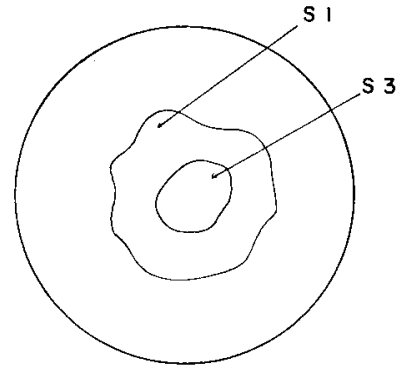

Disque de papier filtre inférieur

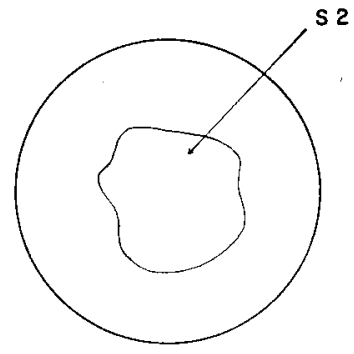

Disque de papier filtre supérieur

FIG. I

\section{Compression}

La méthode utilisée était celle exposée par nous-mêmes (GoutefongeA, 1960), où l'échantillon de viande, placé entre deux disques de papier-filtre au fond d'un cylindre, est comprimé pendant $5 \mathrm{mn}$ par le piston pesant $225 \mathrm{~g}$ que l'on laisse tomber d'une hauteur constante, afin d'avoir des conditions expérimentales reproductibles. 
Pour les 18 premiers porcs, le papier utilisé était conservé dans un dessiccateur à $\mathrm{CaCl}_{2}$. Pour les porcs suivants, il était conservé dans un humidificateur à $\mathrm{K} \mathrm{Cl}$.

Nous avons enregistré :

- la différence des poids initial et final de l'échantillon de viande,

- les surfaces des taches de diffusion du jus sur le disque de papier inférieur $\left(S_{1}\right)$ et supérieur $\left(\mathrm{S}_{2}\right)$,

- la surface de l'échantillon de viande après l'opération $\left(\mathrm{S}_{\mathbf{3}}\right)$.

Les surfaces $S_{1}, S_{2}$ et $S_{3}$ sont représentées figure $r$.

\section{Centrifugation}

Dans un tube de centrifugeuse cannelé de $45 \mathrm{ml}$, à $5 \mathrm{~g}$ de muscle, on ajoute $7,5 \mathrm{mI}$ d'eau. Le broyage est effectué à l'aide d'un microbroyeur Durel et Sausse qui permet de réaliser l'opération dans le tube de centrifugeuse. Les échantillons sont centrifugés pendant $20 \mathrm{mn}$ à $6500 \mathrm{t} / \mathrm{mn}$ dans une centrifugeuse Jouan refroidie par circulation d'eau.

Après la centrifugation, le liquide surnageant est enlevé, les tubes contenant le culot de centrifugation sont mis à égoutter to minutes et on détermine par pesée la perte ou le gain de poids qui correspond à une perte ou à une rétention d'eau. Cette méthode a été appliquée sur les porcs 2 I à 30.

\section{RÉSULTATS}

\section{Variabilité}

Lors d'essais préliminaires, nous avons étudié la variabilité des différentes méthodes, en appliquant la même méthode à ro échantillons d'un même muscle. Nous disposions alors d'un muscle normal $(\mathrm{pH} 24 \mathrm{~h}=6,00)$ et d'un muscle exsudatif $(\mathrm{pH} 24 \mathrm{~h}=5,47)$.

Le pouvoir de rétention d'eau a été exprimé de différentes façons.

Méthode de pression.

$a$ - Perte de poids en p. Ioo de tissu frais.

$b$ - Somme $\mathrm{S}_{1}+\mathrm{S}_{2}$ des surfaces des taches de jus sur les papiers-filtres supérieur et inférieur, en $\mathrm{cm}^{2} / \mathrm{g}$ de tissu frais.

TABLEAU 2

Variabilité des différentes méthodes de mesure du pouvoir de rétention d'eau

\begin{tabular}{|c|c|c|c|c|}
\hline Qualité & \multicolumn{2}{|c|}{ Méthode et mode d'expression des résultats } & Moyenne $\pm \sigma$ & $\begin{array}{c}\text { Coefficient } \\
\text { de variation } \\
(\%)\end{array}$ \\
\hline \multirow[t]{2}{*}{ Exsudatif } & Pression & $\begin{array}{l}\text { Perte de poids en p. } 100 \text { de tissu frais. } \\
\text { Somme des surfaces } S_{1}+S_{2} \text { en } \mathrm{cm}^{2} / \mathrm{g} \text { tissu } \\
\text { frai. } \\
S_{1}+S_{2}-2 S_{3} \text { en } \mathrm{cm}^{2} / \mathrm{g} \text { de tissu frais. }\end{array}$ & $\begin{array}{r}29,03 \pm 2,18 \\
15,39 \pm 1,97 \\
7,45 \pm 1,85 \\
\end{array}$ & $\begin{array}{r}7,51 \\
12,80 \\
24,83\end{array}$ \\
\hline & Centrifugation & Perte de poids en $p .100$ de tissu frais. & $14,86 \pm 0,83$ & 5,61 \\
\hline \multirow[t]{2}{*}{ Normal } & Pression & $\begin{array}{l}\text { Perte de poids en p. } 100 \text { de tissu frais. } \\
\text { Somme des surfaces } S_{1}+S_{2} \text { en } \mathrm{cm}^{2} / g \text { de } \\
\text { tissu frais. } \\
S_{1}+S_{2}-2 S_{3} \text { en } \mathrm{cm}^{2} / g \text { de tissu frais. }\end{array}$ & $\begin{array}{r}12,11 \pm 2,37 \\
8,77 \pm 1,17 \\
1,08 \pm 0,45 \\
\end{array}$ & $\begin{array}{l}19,65 \\
13,34 \\
41.66\end{array}$ \\
\hline & Centrifugation & Gain cle poids en p. 100 de tissu frais. & $4,14 \pm 0,71$ & 17,20 \\
\hline
\end{tabular}


$c$-. Surface obtenue en retranchant de la somme $S_{1}+S_{2}$ ci-dessus le double de la surface $S_{3}$ de l'échantillon de muscle après pression, en $\mathrm{cm}^{2} / \mathrm{g}$ de tissu frais.

Méthode de centrifugation.

- Perte ou gain de poids en p. Ioo de tissu frais.

Les résultats de ces essais sont rassemblés dans le tableau 2.

\section{Comparaison des méthodes}

A partir des données obtenues avec les 480 échantillons, nous avons calculé les coefficients de corrélation, d'une part, entre les résultats obtenus par les différentes méthodes et, d'autre part, entre le pH et les résultats obtenus par chaque méthode.

Les valeurs de ces coefficients sont rassemblées dans les tableaux 3 et 4 .

\section{DISCUSSION}

\section{Répétabilité des mesures}

Dans l'ensemble, les résultats rassemblés dans le tableau 2 montrent une variabilité des mesures assez importante, surtout pour la méthode de pression, lorsque l'on prend en considération la surface $S_{1}+S_{2}-2 S_{3}$.

TABLEAU 3

Coefficients de corrélation entre les résultats obtenus par les différentes méthodes

\begin{tabular}{|c|c|c|c|c|c|}
\hline $\begin{array}{l}\text { No de la } \\
\text { combi- } \\
\text { naison }\end{array}$ & $\mathrm{X}$ & $\mathrm{Y}$ & $\begin{array}{c}\text { Nombre } \\
\text { de porcs } \\
\text { et numéros }\end{array}$ & $\begin{array}{l}\text { Nombre } \\
\text { d'échan- } \\
\text { tillons }\end{array}$ & \\
\hline 1 & $\begin{array}{c}\text { Eau extraite par pression en } \\
\text { p. } 100 \text { de tissu frais }\end{array}$ & $\begin{array}{l}\text { Somme des surfaces } \\
\mathrm{S}_{1}+\mathrm{S}_{2} \text { en } \mathrm{cm} / \mathrm{2}^{\mathrm{g}}\end{array}$ & 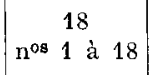 & 288 & $+0,91 \pm 0,01$ \\
\hline 2 & $\begin{array}{l}\text { Eau extraite par pression en } \\
\text { p. } 100 \text { de tissu frais }\end{array}$ & $\begin{array}{l}\text { Somme des surfaces } \\
\mathrm{S}_{1}+\mathrm{S}_{2} \text { en } \mathrm{cm}^{2} / \mathrm{g}\end{array}$ & $\begin{array}{c}12 \\
\text { nos }^{\text {19 }} 19 \text { à } 30\end{array}$ & 192 & $+0,79 \pm 0,03$ \\
\hline 3 & $\begin{array}{c}\text { Eau extraite par pression en } \\
\text { p. } 100 \text { de tissu frais }\end{array}$ & $\mathrm{S}_{1}+\mathrm{S}_{2}-2 \mathrm{~S}_{3} \mathrm{en} \mathrm{cm}^{2} / \mathrm{g}$ & 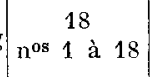 & 288 & $+0,87 \pm 0,02$ \\
\hline 4 & $\begin{array}{l}\text { Eau extraite par pression en } \\
\text { p. } 100 \text { de tissu frais. }\end{array}$ & 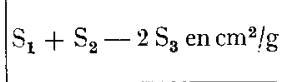 & $\left|\begin{array}{c}12 \\
n^{\text {os }} 19 \text { à } 30\end{array}\right|$ & 192 & $+0,86 \pm 0,02$ \\
\hline 5 & $\begin{array}{l}\text { Eau extraite par centrifugation } \\
\text { en p. } 100 \text { de tissu frais. }\end{array}$ & $\begin{array}{l}\text { Eau extraite par pres- } \\
\text { sion en p. } 100 \text { de tissu } \\
\text { frais }\end{array}$ & 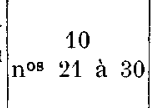 & 160 & $+0,76 \pm 0,03$ \\
\hline 6 & $\begin{array}{l}\text { Eau extraite par centrifugation } \\
\text { en p. } 100 \text { de tissu frais. }\end{array}$ & $\mathrm{S}_{1}+\mathrm{S}_{2}$ en $\mathrm{cm}^{2} / \mathrm{g}$ & 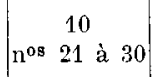 & 160 & $+0,53 \pm 0,05$ \\
\hline 7 & $\begin{array}{l}\text { Eau extraite par centrifugation } \\
\text { en p. } 100 \text { de tissu frais. }\end{array}$ & $\mathrm{S}_{1}+\mathrm{S}_{2}-2 \mathrm{~S}_{3} \mathrm{encm}^{2} / \mathrm{g}$ & 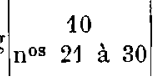 & 160 & $+0,69 \pm 0,04$ \\
\hline
\end{tabular}


On peut également noter que le coefficient de variation est nettement plus élevé, pour ces méthodes, dans le cas du muscle normal. Cette variabilité plus grande est due au fait que les valeurs moyennes sont plus faibles chez le muscle normal. Cette remarque est également valable pour expliquer le coefficient de variation très important des résultats obtenus en prenant en considération la quantité $S_{1}+S_{2}-2 S_{3}$.

Toutefois, bien que les coefficients de variation soient élevés, chaque méthode différencie nettement les 2 échantillons.

\section{Comparaison des méthodes}

Il faut aussi noter que si les résultats obtenus par la méthode de pression représentent toujours une perte d'eau, par contre, ceux obtenus par la méthode de centrifugation représentent, lorsque le $\mathrm{pH}$ est assez élevé (à partir de $\mathrm{pH}$ 6,00 environ), une absorption d'eau. C'est en particulier le cas du muscle normal dans notre expérience préliminaire et, parmi les r6o échantillons qui ont été soumis à cette méthode, 33 ont présenté une rétention d'eau. Les $\mathrm{pH}$ de ces échantillons étaient tous supérieurs à 5,94 .

\section{TABLEAU 4}

Coefficients de corrélation entre les résultats obtenus par les différentes méthodes et le pH

\begin{tabular}{|c|c|c|c|c|c|}
\hline $\begin{array}{l}\text { No de la } \\
\text { combi- } \\
\text { naison }\end{array}$ & $\mathrm{X}$ & $\mathrm{Y}$ & $\begin{array}{c}\text { Nombre } \\
\text { de porcs } \\
\text { et numéros }\end{array}$ & $\begin{array}{l}\text { Nombre } \\
\text { d'échan- } \\
\text { tillons }\end{array}$ & \\
\hline 8 & $\begin{array}{l}\text { Eau extraite par pression en p. } 100 \text { de tissu } \\
\text { frais. }\end{array}$ & $\mathrm{pH}$ & 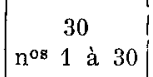 & 480 & $-0,71 \pm 0,02$ \\
\hline 9 & $\begin{array}{l}\text { Eau extraite par pression en p. } 100 \text { de tissu } \\
\text { frais. }\end{array}$ & $\mathrm{pII}$ & 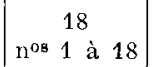 & 288 & $-0,73 \pm 0,03$ \\
\hline 10 & $\begin{array}{l}\text { Eau extraite par pression en p. } 100 \text { de tissu } \\
\text { frais. }\end{array}$ & $\mathrm{pH}$ & $\begin{array}{c}12 \\
\mathrm{n}^{\text {os }} \\
19\end{array}$ & 192 & $-0,73 \pm 0,04$ \\
\hline 11 & $\begin{array}{l}\text { Somme des surfaces } S_{1}+S_{2} \text { en } \mathrm{cm}^{2} / g \text { de tissu } \\
\text { frais. }\end{array}$ & pH & 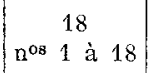 & 288 & $一 0,69 \pm 0,03$ \\
\hline 12 & $\begin{array}{l}\text { Somme des surfaces } S_{1}+S_{2} \text { en } \mathrm{cm}^{2} / g \text { de tissu } \\
\text { frais. }\end{array}$ & $\mathrm{pH}$ & $\begin{array}{ccc} & 12 \\
\text { noв } & 19 & \\
\text { à } & 30\end{array}$ & 192 & $-0,52 \pm 0,05$ \\
\hline 13 & $S_{1}+S_{2}-2 S_{3}$ en $\mathrm{cm}^{2} / g$ de tissu frais. & $\mathrm{pH}$ & $\begin{array}{cccc} & 18 & \\
n^{08} & 1 & a ̀ & 18\end{array}$ & 288 & $-0,89 \pm 0,01$ \\
\hline 14 & $S_{1}+S_{2}-2 S_{3}$ en $\mathrm{cm}^{2} / g$ de tissu frais. & $\mathrm{pH}$ & 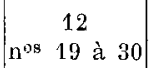 & 192 & $-0,69 \pm 0,04$ \\
\hline 15 & $\begin{array}{l}\text { Eau extraite par centrifugation en p. } 100 \text { de } \\
\text { tissu frais. }\end{array}$ & $\mathrm{pH}$ & $\begin{array}{cc}10 \\
n^{\text {os }} & 21 \text { à } 30\end{array}$ & 160 & $-0,91 \pm 0,01$ \\
\hline
\end{tabular}

Il n'est toutefois pas possible de généraliser car, par cette méthode, des échantillons à $\mathrm{pH}$ supérieur à 6,00 peuvent très bien subir une perte d'eau (nous avons même 
obtenu une perte d'eau, minime certes, avec des échantillons à $\mathrm{pH} 24 \mathrm{~h}$ de 6,34 et $6,56)$. Ceci doit d'ailleurs nous inciter à rechercher si le pouvoir de rétention d'eau est sous la dépendance exclusive du $\mathrm{pH}$ ou s'il s'agit de 2 phénomènes concomitants.

Les coefficients de corrélation entre les différentes méthodes sont tous significatifs à $\mathrm{P}=$ o, or (tableau 3 ).

On remarque une différence assez nette entre les cœfficients des combinaisons I et 2 , I ayant une valeur nettement supérieure. L'emploi du papier-filtre humide (atmosphère đe $\mathrm{KCl}$ saturé) qui a été fait pour les porcs I9 à 30 n'a donc pas apporté d'amélioration, au contraire. Pourtant, les coefficients des combinaisons 3 et 4 sont très voisins. En exprimant les résultats par la quantité $S_{1}+S_{2}=2 S_{3}$ en $\mathrm{cm}_{2} / \mathrm{g}$ de tissu frais, on peut donc dire que le degré de siccité du papier n'a pas d'importance.

Le coefficient de la combinaison 5 montre que la relation entre la méthode de compression et la méthode de centrifugation est assez étroite ; il est toutefois permis de penser que ces deux méthodes ne mesurent peut-être pas exactement la même propriété, l'état physique du muscle étant très différent dans les deux cas.

Les coefficients de corrélation entre les résultats obtenus par les différentes méthodes et le $\mathrm{pH}$ sont également tous significatifs à $\mathrm{P}=0$,or. (Tableau 4 )

Le plus élevé de ces coefficients est celui de la combinaison ${ }_{5}$, ce qui ne signifie pas de façon absolue que la centrifugation donne du pouvoir de rétention d'eau réel du muscle une meilleure représentation que la compression, mais simplement que ce que l'on a mesuré par cette méthode est lié plus étroitement au pH.

\section{CONCI,USION}

L'examen des différentes méthodes proposées nous permet de dégager les conclusions suivantes :

- Il est préférable, lorsque l'on emploie la méthode de pression, d'exprimer les résultats en perte d'eau en p. Ioo du poids frais. Parmi les trois modes d'expression que nous avons utilisés, c'est celui-ci qui a la variabilité la moins grande. D'autre part, l'utilisation de la planimétrie demande plus de temps.

- La méthode de centrifugation présente l'inconvénient, par rapport à la précédente, d'exiger des quantités d'échantillons plus importantes, ce qui peut être parfois gênant. Elle peut être assez rapide ( 16 échantillons à la fois) et la variabilité des résultats est du même ordre de grandeur que celle de la méthode de pression, lorsque que les résultats sont exprimés comme précédemment indiqué.

Ces deux méthodes se prêtent assez bien à l'utilisation en grande série.

Toutefois, ces deux méthodes sont assez grossières et si elles sont suffisantes pour des déterminations courantes, il nous semble nécessaire d'avoir une autre méthode à notre disposition.

Cette méthode, qui aurait l'avantage de donner des résultats représentatifs de l'état réel de l'eau dans le muscle, consiste à étudier les phénomènes de sorption et de désorption de l'eau par le muscle. Sa lenteur ne permet pas de l'utiliser en grande série, mais elle pourrait servir de méthode de référence par rapport à laquelle on pourrait tester des méthodes plus rapides.

Reçu pour publication en mars 1963.

Annales de Zootechnie. - I 963 . 


\section{SUMMARY}

DIFFERENT METHODS FOR MEASURING TIE WATER-BINUING CAPACITY OF PORK MLSCLE : a Comparative study. RElation to the MUSCle pH.

Two methods for measuring the water-binding capacity of pork muscle were compared in a study of 480 samples from 30 Large-Jhite animals. The first one was a pressure method, the other one used centrifugation.

The different ways of obtaining the pressure method results are compared. The relations of the $\mathrm{pH}$ to the water binding capacity, as measured by the different methods are studied.

The centrifugation and the pressure methods (the weight loss being related to the freshweight) have about the same variability. and the correlation coefficient between them is highly significant.

The centrifugation results were better correlated with the $\mathrm{pH}$ than the pressure results.

\section{RÉFÉRENCES BIBLIOGRAPHIQUES}

Briskey l. J., Bray R. W., Hoekstra W. G., Phillips P. H., Grliner R. H., I959. The chemical and physical characteristics of various pork ham muscles classes. J. Anim. Sci., 18, I 4 6- I 52.

Briskey E. J., Hoekstra W. G., Bray R. W., Grummer R. H., i 960 . A comparison of certain physical and chemical characteristics of eight pork muscles. J. Anim. Sci 19, 214-225.

Goutefongen R., ig6o. Description d'un nouvel appareil pour mesurer le pouvoir de rétention d'eau de la viande. VIth Meeting of Meat Research Institutes. Uirecht, 1960.

Grat R., Hamn R., 1952. Eine einfache Methode zur Bestimmung der Wasserbindung im Fleisch. Fleishwirtschaft, 4, 295 .

GRaU R., Ham R., 1953. Eine einfache Methode zur Bestimmung der Wasserbindung im Muskel. Naturwissenschaften, $\mathbf{4 0}, 29-30$.

Grau R., Hamm R., 1956. Die Bestimmung der Wasserbindung des Fleisches mittel der Press methode. Fleischwirtschajt, 8, 733-736.

Hamburger J., Matine G., 1952. Métabolisme de l'ean (36). Editions médicales, Flammarion. Paris.

NinnvaARa F. P., Pohja M. S., I 954. Zur Theorie der Wasserbindung des Fleisches. Fleischwirtschaft, 6, 192-193.

Schon L., STOSIEK M., I958. Untersuchungen zum Salthaltungsvermögen im MuskelHeish von Rindern. Fleischwirlschatt, 11, $769-770$.

Sherman P., 196r. The water binding capacity of fresh pork. Food Technol., 15, 79-87.

SwifT C. E., Berman M.D., I959. Factors affecting the water retention of beef. Food Technol., 13, 365-370.

URBin M. C., Zessin D.A., Wilson G. D., ig62. Observations on a method of determining the water bindding properties of meat. J. Anim. Sci., 21, 9-1S.

Wierbicki F., Kunkte L.E., Dentierage F.E., 1957. Changes in the water holding capacity and cationic shifts during the heating and freezing and thawing of meat revealed by a simple centrifugal method for measuring skrinkage. Food Technol., 11, 69-73.

Wierbicki E., DeAtherage F. E., 1958. Determination of water holding capacity of fresh meat. J.Agr. Food Chem., 6, 387-392.

Wismer-Pedersex J., I958. Quality of pork in relation to rate of $\mathrm{pH}$ change post-mortem. 4th leeting of Meat Research Institutes. Cambridge., 1958. 